\title{
Diagnostic Auscultatory Complex in Coarctation of the Aorta
}

\author{
GEORGE A. BOUSVAROS * \\ From the foint Cardiorespiratory Service, The Royal Victoria Hospital, Montreal, Canada
}

In spite of numerous descriptions of the auscultatory and phonocardiographic findings in "adult" coarctation of the aorta (Reifenstein, Levine, and Gross, 1947; Wells, Rappaport, and Sprague, 1949; Brown et al., 1959; Cleland et al., 1956; Wood, 1956; Spencer, Johnston, and Meredith, 1958; Gasul, Arcilla, and Lev, 1966), no specific diagnostic auscultatory pattern has been outlined. The general conclusion has been that "auscultation is relatively unimportant in establishing the diagnosis of coarctation ..." (Gardiner, 1959).

The purpose of this report is to draw attention to an auscultatory complex which appears diagnostic of coarctation. This consists of the combination of an early systolic ejection sound (click) with a midlate systolic murmur (Fig. A-D) audible at the lower left sternal border and the apex. These two auscultatory signs have been noted before in coarctation but the diagnostic specificity of their association has not been recognized. The ejection click (Leatham and Vogelpoel, 1954; Cleland et al., 1956; Wood, 1956; Gasul et al., 1966; Hancock, 1966) may be immediately followed by an ejection systolic murmur of varying length. In other cases, however, it is followed, after a gap of at least 0.04 sec., by a mid-late systolic murmur. Such a murmur (Reifenstein et al., 1947; Wells et al., 1949; Wood, 1956; Spencer et al., 1958; Segal and Likoff, 1964; Gasul et al., 1966) is produced by flow either through collateral vessels or through the constricted segment of the aorta, and its delayed onset is due to the time taken for propagation of the pulse wave along the arterial system.

The association of an early systolic click with a delayed systolic murmur is not encountered in any

Received July 19, 1966.

* Present address: Veterans Administration Hospital, Albany, New York, U.S.A. other disease except in coarctation. An ejection click due to such causes as stenosis or insufficiency of the pulmonary or aortic valve, idiopathic dilatation of the pulmonary artery, or pulmonary hypertension is commonly accompanied by an ejection systolic murmur with an early onset. Likewise, apart from coarctation, late systolic murmurs are not associated with early systolic clicks. Such murmurs are commonest in mild mitral incompetence (Barlow et al., 1963; Segal and Likoff, 1964) when they may occasionally start with midlate clicks. Delayed systolic murmurs also occur in pulmonary branch stenosis (Bousvaros and Palmer, 1965) and sometimes in idiopathic hypertrophic subaortic stenosis (Segal and Likoff, 1964). In the latter condition the murmur, rarely, has an explosive onset, simulating a click which, however, is not early but coincides with the peak of the percussion wave of the carotid sphygmogram (Hancock, 1966).

The auscultatory complex described here is not present in all patients with coarctation, but is not uncommon either, as it was encountered in 13 of 27 cases personally observed since 1960 . It appears to be sufficiently specific and it should become a valuable complement to the difference in pulses between upper and lower limbs in establishing the diagnosis of "adult" coarctation of the aorta.

\section{SUMMARY}

The auscultatory signs of coarctation of the aorta have previously been considered evocative, but not specific of the diagnosis. The present report suggests that the association of an early systolic click with a delayed systolic murmur is encountered only in coarctation of the aorta. This diagnostic complex was present in 13 of a personally observed group of 27 patients. It should become a 


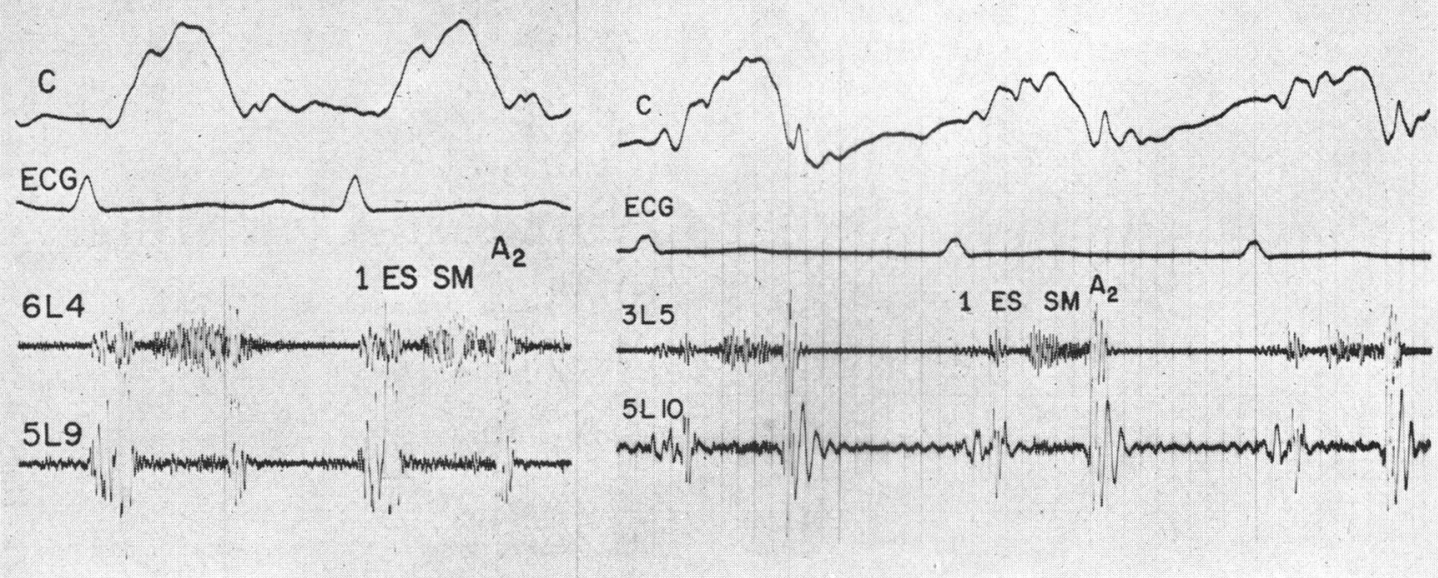

A

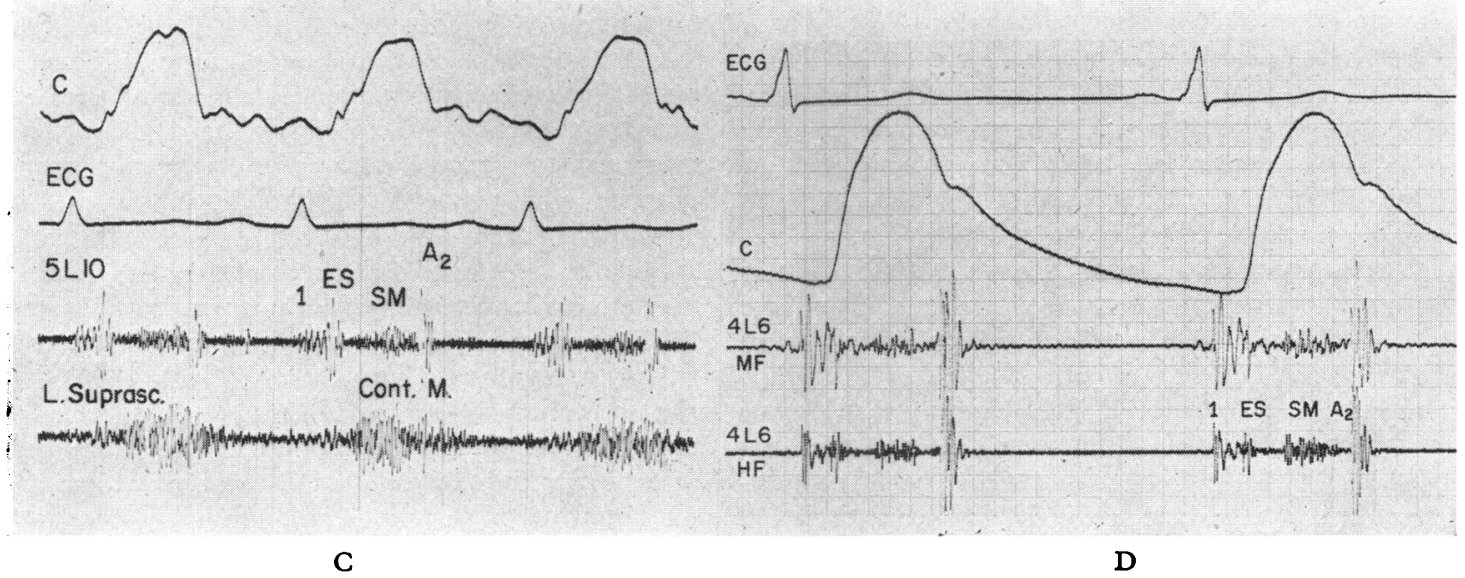

Phonocardiograms of four patients with adult coarctation illustrating the described complex. The systolic click is early coinciding with the onset of the carotid sphygmogram. The systolic murmur starts 0.04 to $0.08 \mathrm{sec}$. after the click. In $A$ and $B$ it is louder at the left sternal border than at the mitral area. In $A$ the murmur spills beyond the second sound, presumably due to the delay in propagation of the arterial pulse wave to the periphery. In $\mathrm{C}$ the murmur is best shown at the mitral area; the second microphone records a continuous murmur at the left suprascapular area. In $D$ both sound tracings represent two different (medium and high) frequency bands from the same microphone. Time lines: $0.04 \mathrm{sec}$. apart in A, B, and C, and $0.05 \mathrm{sec}$. between thick lines in panel D. Abbreviations: $C=$ carotid sphygmogram, ECG $=$ electrocardiogram, $3 \mathrm{~L} 5=$ third left interspace $5 \mathrm{~cm}$. from midline etc., $\mathrm{HF}=$ high frequency band, $M F=$ medium frequency, $1=$ first sound, ES = ejection sound, $S M=$ systolic murmur, Cont. $M=$ continuous murmur, $\mathrm{A}_{2}=$ aortic element of second sound.

valuable complementary sign in the diagnosis of this condition.

I am indebted to Dr. D. C. Deuchar for permission to include cases and phonocardiograms obtained while I was working at Guy's Hospital, and to Dr. Maurice McGregor for reviewing the manuscript.

\section{REFERENCES}

Barlow, J. B., Pocock, W. A., Marchand, P., and Denny, M.
(1963). The significance of late systolic murmurs. Amer. Heart F., 66, 443.

Bousvaros, G., and Palmer, W. (1965). Phonocardiographic features of the systolic murmur in pulmonary artery stenosis. Brit. Heart f., 27, 374.

Brown, C. J. O., Deans, B. L., Gardiner, J. M., Jackson, A. V., Kay, H. B., and Morris, K. N. (1959). Congenital heart disease. III. Coarctation of the aorta. Med. F. Aust., 1, 857.

Cleland, W. P., Counihan, T. B., Goodwin, J. F., and Steiner, R. E. (1956). Coarctation of the aorta. Brit. med. F., 2, 379. 
Gardiner, J. M. (1959). The phonocardiogram in congenital heart disease. Aust. Ann. Med., 8, 225.

Gasul, B. M., Arcilla, R. A., and Lev, M. (1966). Heart Disease in Children. Diagnosis and Treatment, p. 905. J. B. Lippincott, Philadelphia and Montreal.

Hancock, E. W. (1966). The ejection sound in aortic stenosis. Amer. F. Med., 40, 569.

Leatham, A., and Vogelpoel, L. (1954). The early systolic sound in dilatation of the pulmonary artery. Brit. Heart $\mathcal{F}$., 16, 21.

Reifenstein, G. H., Levine, S. A., and Gross, R. E. (1947). Coarctation of the aorta. A review of 104 autopsied cases of the "adult type", 2 years of age or older. Amer. Heart F., 33, 146.

Segal, B. L., and Likoff, W. (1964). Late systolic murmur of mitral regurgitation. Amer. Heart f., 67, 757.

Spencer, M. P., Johnston, F. R., and Meredith, J. H. (1958). The origin and interpretation of murmurs in coarctation of the aorta. Amer. Heart F., 65, 722.

Wells, B. G., Rappaport, M. B., and Sprague, H. B. (1949). The sounds and murmurs in coarctation of the aorta. Amer. Heart f., 38, 69.

Wood, P. (1956). Diseases of the Heart and Circulation, 2nd ed., p. 335. Eyre and Spottiswoode, London. 